\title{
IMPACT-INDUCED ACTIVITY OF THE ASTEROID-COMET P/1996N2 ELST- PIZARRO: YES OR NO?
}

\author{
H. BOEHNHARDT \\ University Observatory \\ Scheinerstr. 1, D-81679 München, Germany \\ European Southern Observatory \\ Alonso de Cordova 3107, Santiago, Chile \\ Z. SEKANINA \\ Jet Propulsion Laboratory \\ California Institute of Technology \\ 4800 Oak Grove Drive, Pasadena CA 91109, USA \\ A. FIEDLER \\ University Observatory \\ Scheinerstr. 1, D-81679 München, Germany \\ H. RAUER \\ DLR, Institute for Planetary Exploration \\ Rudower Chaussee 5, D-12484 Berlin, Germany \\ R. SCHULZ \\ European Space Agency \\ Keplerlaan 1, NL-2200 AG Noordwijk, The Netherlands \\ AND \\ G. TOZZI \\ Osservatorio Astrofisico di Arcetri \\ Largo E. Fermi 5, I-50125 Firenze, Italy
}

\begin{abstract}
P/1996 N2 Elst-Pizarro was discovered with a tail of dust in July 1996 and was thus classified as periodic comet. From the orbit point of view it belongs to the outer asteroid belt and is very likely a member of the Themis family, a group of bodies originating from an asteroid collision.

The absence of a coma and the presence of the needle-like anti-tail in August 1996 did not exclude the possibility of a sudden emission event which could have been caused by the impact of another minor body on the object. However, with the development of a normal tail between September and November 1996 it became obvious that the dust emission episode continued at least for 2 months after perihelion, a scenario that is typical for gas-driven cometary activity. However, it is still possible that a collision triggered the event.

The paper summarizes the observational pro and cons for impact-induced activity in this object, possibly the first ever detected comet-asteroid of the solar system.
\end{abstract}

\section{The Object Type: Asteroid or Comet?}

P/1996N2 Elst-Pizarro was discovered by Eric Elst on 7 August 1996 on 1-meter Schmidt telescope exposures taken on 14 and 16 July 1996 by Guido Pizarro at the European Southern Observatory ESO at La Silla, Chile (Elst and Pizarro, 1996). At the time of discovery as well as during the subsequent $11 / 2$ months the object exhibited a straight and narrow, several arcmin long tail pointing almost toward the Sun (Pravec, 1996). Although no discernible coma was reported from the follow-up observations made in August 1996 (Offut, 1996), the object was classified by the 
Central Bureau for Astronomical Telegrams as a comet, mainly because of the continuous presence of the tail (Marsden, 1996a).

TABLE 1. Comparison of Orbital Elements of Elst-Pizarro with average elements of the Themis Family

\begin{tabular}{lcc}
\hline & Elst-Pizarro & Themis Family \\
\hline semi-major axis (AU) & 3.156 & $\mathbf{3 . 1 4 6}$ \\
eccentricity & 0.167 & 0.168 \\
inclination (deg) & $\mathbf{1 . 3 8}$ & 1.87 \\
reference & Marsden, 1996b & Kresák, 1971 \\
\hline
\end{tabular}

However, as already noted by Marsden (1996b) after the initial orbit determination, the orbit of $\mathrm{P} / 1996 \mathrm{~N} 2$ Elst-Pizarro is typical as a main belt asteroid rather than a comet. Moreover, astrometric observations from 1979 and 1985 show these to be earlier detections of P/1996N2 Elst-Pizarro reported to be asteroid (Marsden, 1996b,c; McNaught \& Hawkins 1996). Hence, P/1996N2 ElstPizarro could be a cometary object in the main asteroid belt and thus represent the first cometasteroid ever detected. However, one should exercise a caution since the early 1996 observations did not unambiguously support the comet classification, e.g. no coma was found and no spectrum was available which could be used for a search of emission features typical for comets. Furthermore, there exists a remarkable coincidence between the semi-major axis, eccentricity and inclination of P/1996N2 Elst-Pizarro and those of the Themis asteroid family (see Table 1) suggesting that $\mathrm{P} / 1996 \mathrm{~N} 2$ Elst-Pizarro is possibly to be a member of this family. This family contains many C-type and some B- and F-type asteroids of typically low geometric albedo (0.01-0.06). Their members are likely to consist of primitive and metamorphic grades of carbonoceous chondritic material originating from a uniform CM-like parent body (Gradie et al., 1979; Chapman et al., 1989).

\section{Collision-Induced Origin for the Tail?}

The enigmatic early reports of a comet in the asteroid belt showing a tail without a coma led some people to speculate about a possible impact-induced origin of the tail (Balam, 1996). Furthermore, an initial analysis of the narrow sunward tail seen in August 1996 (Sekanina, 1996) did not exclude the possibility of a brief emission episode of micron to sub-micron size dust which should have taken place between 40 to 80 days after perihelion (which occurred on 16 April 1996). FinsonProbstein calculations of the tail geometry predicted the possibility of a second, "normal" dust tail pointing essentially away from the Sun (Boehnhardt, 1996). The scenario applied to the period from September to December 1996 in case the emission episode lasted fairly long. the normal tail was indeed first observed around mid September 1996 (Bochnhardt et al., 1996).

\subsection{OBSERVATIONAL FACTS}

In the following we report and summarize the preliminary results of our observations of $\mathrm{P} / 1996 \mathrm{~N} 2$ Elst-Pizarro collected between 23 August and 5 November 1996 at ESO La Silla, Chile. These target-of-opportunity observations were performed in the context of a joint programme of European scientists in support of the ROSETTA and ISO missions of the European Space Agency (ESA). They involved broadband BVR imaging and low dispersion spectroscopy (spectral range about 350 $-700 \mathrm{~nm}$, spectral resolution about $0.8 \mathrm{~nm} / \mathrm{pixel}$ ) in the visible wavelength range with the focal reducers DFOSC at the Danish 1.5 meter telescope and EFOSC2 at the MPG/ESO 2.2 meter telescope.

When corrected for contributions from the dust tails (sce below), the object's head in the available set of images appears to be stellar. This is concluded from a comparison of cross-cut profiles through the seeing disc of the nucleus with point-spread functions obtained from stars in the same images. The $R$ band brightness of the mucleus of 19.1 mag corresponds to an equivalent radius of $2 \cdots 3.5 \mathrm{~km}$ depending on the geometric albedo assumed $(0.1 \cdots 0.04)$. In co-added frames with a total integration time of more than $60 \mathrm{~min}$, no coma is detected. In late August 1996 the 
straight sunward dust tail had a width of less than 7 arcsec, but extended well beyond the edge of the field of views of our instruments, i.e. it was longer than 8 arcmin which is more than 550000 $\mathrm{km}$ in projection at the "comet". The "normal" tail was pointing in approximately anti-sunward direction from mid-September to early November 1996. It showed a much shorter extension of less than 25 arcsec and appeared to be somewhat broader than the anti-tail (although still less than about 10 arcsec in width). The co-added images and the seeing disc analysis revealed the existence of a very weak and diffuse bridge of light between the normal tail and the anti-tail, which was best seen - though very faint - in October and November 1996, when the plane of the tail was inclined most to the direction from Earth (about $0.6 \mathrm{deg}$ ). A preliminary assessment of the spectra obtained on 23 August (with DFOSC) as well as on 9 September 1996 (with EFOSC2) revealed essentially reflected sunlight without superposed emission band features typical for comets.

Our observational results show that during our monitoring period P/1996N2 Elst-Pizarro exhibited dust tail phenomena without a parallel detectable coma activity.

\subsection{DUST TAIL MODELLING}

During our dust tail analysis of P/1996N2 Elst-Pizarro Monte-Carlo simulations were run for various dates of our observations. The models were compared with the images complemented by the measurements of the tail extension and geometry, i.e. with the position angles, the length and the width of both tails. As already mentioned by Boehnhardt et al. (1996), the simultaneous presence of the normal tail and the anti-tail in September 1996 argues for a dust production which continued for almost two months after perihelion passage. To be more specific, from the measured position angles of both tails it can be concluded that the emission episode producing the dust tails lasted from about 25 May to about 15 July 1996, i.e. from roughly 5 weeks post-perihelion to the time of discovery. The normal tail contained younger dust grains, with a low solar radiation pressure acceleration of less than 0.1 the solar attraction, while the anti-tail corresponds to an early part of the emission episode, with dust grains affected by larger radiation pressure. From the narrow width of the anti-tail and the overall geometry of both tails an expansion velocity of the grains of less than $2.5 \mathrm{~m} / \mathrm{s}$ is inferred.

\section{Conclusions and Speculations}

Based upon the observational evidence and the modelling we conclude that both tails are not likely direct products of an impact event involving the comet-asteroid P/1996N2 Elst-Pizarro shortly after perihelion. Also ruled out can be the interpretation of the dust tails as trails of large dust particles ejected long ago. On the other hand, an interpretation in terms of normal cometary activity suffers clearly from the nondetection of a coma around the object. Also, the inferred expansion velocity is barely above the estimated velocity of escape from the object and considerably lower than typical ejection velocities for the microscopic dust grains whose existence in the tails is implied by the derived radiation pressure accelerations. A plausible scenario for the dust-emission episode may need to invoke an impact as a triggering mechanism that disturbed the surface layer and brought about a temporally limited activity of "icy dirt", a mixture of dust and ice in which the mass of dust dominated by orders of magnitude. Such activity could conceivably explain the extremely low expansion velocity observed. Gentle lifting or disintegration processes for dust may also be considered as potential explanation scenarios, but require a thorough modelling and comparison with the observations. And, of course, further observational monitoring and the analysis of possibly existing data covering the emission period of the object from May to July 1996 will be very useful to solve the two existing major questions about $\mathrm{P} / 1996 \mathrm{~N} 2$ Elst-Pizarro: the object type and the nature of the activity in 1996. For both issues a comparitive study with another intermediate candidate between comets and asteroids, i.e. 107P/Wilson-Harrington (McFadden et al. 1994), may be of great interest.

\section{References}

Balam, D. (1996) Asteroid or Comet?, Sky and Telescope 92, p. 10

Bell, J.F, Davis, D.R., Hartmann, W.K., Gaffey, M.J. (1989) Asteroids: The Big Picture, Asteroids II (eds. R.P. Binzel, T. Gehrels, M. Shapley Matthews), Univ. Arizona Press, Tucson, pp. 921-945

Boehnhardt, H. (1996) Comet P/1996 N2 (Elst-Pizarro), IAU Circular 6473 
Boehnhardt, H., Schulz, R., Tozzi, G., Z. Sekanina (1996) Comet P/1996 N2 (Elst-Pizarro), IAU Circular 6495

Chapman, C.R., Paolicchi, P., Zappala, V., Binzel, R.P., Bell, J.F. (1989) Asteroid Families: Physical Properties and Evolution, Asteraids II (eds. R.P. Binzel, T. Gehrels, M. Shapley Matthews), Univ. Arizona Press, Tucson, pp. 386-415

Elst, E., Pizarro, G. (1996) Comet P/1996 N2 (Elst-Pizarro), IA U Circular 6456

Gradie, J.C., Chapman, C.R., Williams, J.G. (1979) Families of Minor Planets, Asteroids (ed. T. Gehrels), Univ. Arizona Press, Tucson, pp. 359-390

Kresák, L. (1971) Orbital Selection Effects in the Palomar-Leiden Asteroid Survey, Physical Studies of Minor Planets (ed. T. Gehrels), NASA SP-267, Washington DC, pp. 197-210

Marsden, B.G. (1996a) Comet P/1996 N2 (Elst-Pizarro), IAU Circular 6456

Marsden, B.G. (1996b) Comet P/1996 N2 (Elst-Pizarro), IAU Circular 6457

Marsden, B.G. (1996c) Comet P/1996 N2 (Elst-Pizarro), Minor Planet Electronic Circular 1996-R07

McFadden, L.A. (1994) The Comet-Asteroid Transition: Recent Telescopic Observations, Asteroid, Comets, Meteors 1993 (eds. A. Milani, M. Di Martino, A. Cellino), Kluwer Academic Publishers, Dordrecht, pp. 95-110

McNaught, R.H., Hawkins, M.R.S. (1996) Comet P/1996 N2 (Elst-Pizarro), IAU Circular 6473

Offut, W. (1996) Comet P/1996 N2 (Elst-Pizarro), IAU Circular 6456

Pravec, P. (1996) Comet P/1996 N2 (Elst-Pizarro), IAU Circular 6459

Sekanina, Z. (1996) Comet P/1996 N2 (Elst-Pizarro), IA U Circular 6459 